\title{
Editorial
}

\section{A new Guide for Authors: a clearer path to publication}

We made the most recent changes to our Instructions to Authors in June 2012 by emphasizing the requirement for adherence to the World Medical Association (WMA) Declaration of Helsinki principle of registering clinical trials [1]. It is timely that with the first issue in 2015 (Volume 9, Number 1) that we update our instructions and provide a comprehensive revision of our guidelines. We have amalgamated our Instructions to Authors and Ethical Guidelines as a new Guide for Authors to provide greater clarity regarding ethical issues and our editorial policies. We now provide a greater level of detail describing the various elements of most manuscripts to guide authors composing a manuscript, paying special attention to the presentation of data, including that in Tables and Figures. We hope that the new Guide will provide greater publication integrity by raising the awareness of our authors and reviewers to internationally recognized requirements specified in our Guide, and lead to improvement of manuscripts submitted to us and articles published by us. In this Editorial we highlight some of the important points in our new Guide for Authors.

We recognize that research that advances the development of appropriate treatments and disease prevention measures is important, especially for people in the developing world, in which parts of Asia remain, because many of its inhabitants suffer from poor health and reduced life expectancy. However, factors including a lack of adequate resources, weak infrastructure, local needs and the cultural context, laws and regulations, and administrative arrangements in developing countries require that special considerations be made to apply effectively the fundamental ethical principles that should guide the conduct of all biomedical research involving human subjects, as stated in the WMA Declaration of Helsinki (as adopted more than half a century ago in 1964, and its subsequent revisions, importantly in 1975, and most recently in 2013) [2]. The special considerations that need to be made when applying the Declaration of Helsinki to research undertaken in developing countries are detailed by the Nuffield Council on Bioethics in their discussion papers [3, 4], in the guidelines from the Council for International Organizations of Medical Sciences (CIOMS) in collaboration with the World Health Organization [5], and in the opinion of the European Group on Ethics in Science and New Technologies [6]. The Nuffield Council on Bioethics concluded that research in developing countries is crucial, but must be subject to rigorous ethical safeguards to prevent the exploitation of research participants, whether by corporations, or individuals seeking to further their careers. We seek direction from the guidelines mentioned.

A sound ethical framework provides an essential safeguard to protect and prevent possible exploitation of research participants and patients. At Asian Biomedicine, we understand that applying the principles of the Declaration of Helsinki in practice is often difficult. Some of the principles establish standards that may be very difficult to follow in a developing country setting. These standards may include obtaining consent in emergency settings and providing appropriate care for control groups in vaccine trials. Complete adherence to some of the ideals within the Declaration is sometimes unachievable. However, all guidance recognizes principles such as the requirement for individual informed and documented consent to participate in research as universally accepted. We note that Paragraph 10 of the Declaration states: "Physicians must consider the ethical, legal and regulatory norms and standards for research involving human subjects in their own countries as well as applicable international norms and standards. No national or international ethical, legal or regulatory requirement should reduce or eliminate any of the protections for research subjects set forth in this Declaration" [2]. Some might see the Declaration as aspirational in its aims, promoting ideals that cannot be completely attained by all in all circumstances. Nevertheless, the preeminent principles regarding the ethics of research related to health care as stated in the Declaration of Helsinki are more universally accepted than simply being aspirational ideals, and are essential in setting a sound ethical framework. It behoves all investigators and editors dealing with human subjects to be familiar with them.

Where research ethics committees or institutional review boards (IRBs) that review research ethics are under-resourced or even absent, as is sometimes the case in developing countries, researchers may find it difficult to obtain guidance, or are perhaps ignorant of 
what may be ethically appropriate. The guidelines of a number of national and international bodies, including the Declaration of Helsinki [2] and other specific guidelines on the ethics of health care-related research [7-9], may be used. All research submitted to Asian Biomedicine for publication should at least comply with the current revision of the Declaration of Helsinki and International Council of Medical Journal Editors (ICMJE) recommendations.

There is global consensus in the guidance to the principles of the Declaration of Helsinki that, with few exceptions, informed consent must be obtained from potential research participants before they are included in a study [1-6] as consistent with recommendations of the ICMJE [7], which we endeavor to follow, and U.S. Department of Health and Human Services regulations for the protection of human subjects (45 CFR part 46) [8]. The CIOMS guidelines for individual informed consent are as follows: "For all biomedical research involving humans the investigator must obtain the voluntary informed consent of the prospective subject or, in the case of an individual who is not capable of giving informed consent, the permission of a legally authorized representative in accordance with applicable law" [5]. Clearly, this consent must be documented for this guideline to be effective. Investigators must inform individuals giving consent not only of the possible benefits for themselves and others, but also of the potential risks of participating in research. For research involving children (minors) or individuals without the mental capacity to give consent, a person legally authorized to do so on their behalf must give consent. However, if minors are old enough to understand the proposed research, its potential risks and possible benefits, and the role expected of them as participants, then is it is also desirable to obtain their assent [8]. Assent means an expression of willingness to participate in research by individuals who are by legal definition too young to give informed consent.

Community consent or consent from others may also be required, but should never substitute for individual consent. "In some cultures an investigator may enter a community to conduct research or approach prospective subjects for their individual consent only after obtaining permission from a community leader, a council of elders, or another designated authority. In some communities it is customary for male members of the family to make decisions on behalf of wives and children. Such customs should be respected. However, there will often be a tension between the duty of the researcher to be sensitive to cultural differences, and the duty to ensure that each individual has consented to participate in research. In no case, however, may the permission of a community leader or other authority substitute for individual informed consent." "Before requesting an individual's consent to participate in research, the investigator must provide the following information, in language or another form of communication that the individual can understand." "Consent may be indicated in a number of ways. The subject may imply consent by voluntary actions, express consent orally, or sign a consent form. As a general rule, the subject should sign a consent form, or, in the case of incompetence, a legal guardian or other duly authorized representative should do so" [5].

Questions sometimes arise about what form of documentation of informed consent is appropriate for use in communities where many lack literacy. Some, including the European Union, specify illiteracy as a necessary condition for permitting verbal consent. It may be inappropriate to ask participants to sign consent forms in such situations, and people in some cultures may view signing forms to have sinister implications. Witnessed and documented verbal consent might be used instead. Documentation might include consent that is recorded electronically, e.g. on tape or as a sound file, and then it is important to ensure that the recording is safely archived. "Waiver of informed consent is to be regarded as uncommon and exceptional, and must in all cases be approved by an ethical review committee.” "Investigators should never initiate research involving human subjects without obtaining each subject's informed consent, unless they have received explicit approval to do so from an ethical review committee. However, when the research design involves no more than minimal risk and a requirement of individual informed consent would make the conduct of the research impracticable (for example, where the research involves only excerpting data from subjects' records for epidemiological studies), the ethical review committee may waive some or all of the elements of informed consent" [5], provided that there is protection of confidentiality. Obtaining genuine freely given informed consent requires investigators strive to communicate as accurately as patients, volunteers, or their legal guardians or representatives, can understand about procedures and risks, and to react to their capacity to understand and 
process the information given. A number of circumstances can undermine the apparent genuineness of consent, including coercion, deception, manipulation, deliberate misdescription of what is proposed, lack of disclosure of material facts, or conflicts of interest.

While reviews of research protocols by an ethics review committee or IRB will usually mitigate ethical concerns about research and IRBs will require data to be deidentified if possible, case reports and clinical observations do not always fall under the scope of IRBs; therefore, it is important for medical practitioners to protect the privacy of their patients when publishing case reports. While data that may identify patients are removed easily from laboratory tests, pathology results, and self-reported surveys; photographs of patients are not so easily deidentified, and detailed descriptions of patients are often essential for reports to be complete, clinically meaningful, and educational. Before submitting case reports or a case series to Asian Biomedicine, authors must be obtain documented informed consent from patients (or parents, or legally authorized guardians or representatives in the case of minors or incapacitated adults), whether or not the patients are apparently identifiable.

Photographs of the head that include an eye, ear, a lesion, mole, nevus, or hair or may not apparently disclose the subject's identity to a reader, but a true test of anonymity is whether the patient could recognize their own image. This test of complete anonymity can difficult to pass. A patient's identity might be revealed by a tattoo, jewelry, clothing, fancy nail polish, scars, a nevus, mole, or nipple included in a photograph. In the past, attempts to deidentify patients included masking, usually by placing bars or pixelating the areas over the eyes. However, this practice was abandoned at least 20 years ago because it is not effective in concealing identity [10-14]. Now the criterion standard is judicious cropping to remove identifying areas of the image, while retaining the clinical features to be illustrated. Nevertheless, complete anonymity can be difficult to achieve. Where there is any doubt that patient anonymity can be guaranteed, authors must ask specific permission from the patient to publish their photographs and other identifying information, including on the Internet.

The ICJME determined that any identifying information, such as photographs, pedigrees, and written descriptions, should not be published unless essential to the clinical message or scientific purpose of the article and the patient (or parent, or legal guardian or representative as appropriate) gives written informed consent for publication [7, 14]. Health care professionals should obtain written informed consent before taking any patient photographs as a matter of course, even if there is no initial intent to publish the images. The consent should ideally include permission to have the image published in a medical journal, including an online version. The permission must also give the patient the opportunity to review the manuscript including any photographs, or waive this right.

In line with publications by the Journal of the American Medical Association network and the British Medical Journal's collection, Asian Biomedicine provides an informed consent form for the publication of data regarding any patients who are potentially identifiable in text descriptions, pedigrees, or photographs (available in several languages used in Asia from the managing editor). For various reasons, some authors perceive the requirement for patient consent as an unnecessary burden. However, by waiting until the manuscript is submitted or has been rejected by other journals, it may be even more burdensome to locate and contact patients. Furthermore, if the patient dies, then the author will need to ask their next of kin or authorized legal representative to sign the informed consent, which may be difficult. Standard institutional informed consent forms, such as those used for surgical procedures, incorporating statements regarding photographs cannot always be relied on. We note that these standard permission forms frequently have omissions (such as the important clause about publication of photographs on the Internet). The permission form must state that the permission as irrevocable. Once an article about a patient is published, the identifying information and material cannot be unpublished. Social media may allow widespread dissemination of any information or images. Whenever an author has any doubt whatsoever regarding the preservation of a patient's privacy and anonymity, they should act conservatively and endeavor to obtain appropriate consent. Completion of an informed consent form for patient clinical photographs and other identifying material to be included in Asian Biomedicine may seem like a burden, but this requirement is there to protect the interests of all involved [15]. 
Whereas our previous Instructions to Authors were concerned mainly with recommendations for manuscript preparation and submission, the revised Guide for Authors additionally describes the editorial procedure including the peer review process for all submitted manuscripts, and our editorial policies regarding research integrity, including authorship, conflict of interest, and copyright that were previously presented in our Ethical Guidelines, albeit scantily. By publishing the new Guide for Authors, we hope to clarify our ethical policies and requirements to all our potential authors. To assist authors with compliance to our requirements, we provide an Authors' Statement form available from our managing editor. This Authors' Statement contains declarations regarding the authorship and originality of the submitted work, research ethics, patient privacy and confidentiality, and copyright transfer.

Finally, Asian Biomedicine has adopted the ICMJE Form for Disclosure of Potential Conflicts of Interest. From the time of this issue, we request all authors to fill out the "ICMJE Form for Disclosure of Potential Conflicts of Interest" for each submitted manuscript, and provide them to the corresponding author. For authors where formal agreements for representation allow it, it is sufficient for the corresponding author to sign a Conflicts of Interest Statement on behalf of all authors.

The Editors at Asian Biomedicine respect and appreciate ethical research, indeed when a manuscript contains all required elements, as noted on the Manuscript checklist in our new Guide for Authors, and statements of ethics compliance, it makes our job easier. We hope that our authors and readers will appreciate the improved clarity of our new Guide. It is our ambition to become one of the leading biomedical journals in the Asian region. We consider that the best way to achieve this goal is by continuing improvement of publication integrity and editorial quality.

\section{References}

1. Can we do better at registering clinical trials? Asian Biomed. 2013; 6:333-5 [editorial].

2. World Medical Association Declaration of Helsinki: ethical principles for medical research involving human subjects. JAMA 2013; 310: 2191-4.

3. Nuffield Council on Bioethics. The ethics of research related to healthcare in developing countries [online]. 2002. [cited 2015 Jan 16]; Available from: http:// nuffieldbioethics.org/wp-content/uploads/2014/07/ Ethics-of-research-related-to-healthcare-indeveloping-countries-I.pdf

4. Nuffield Council on Bioethics. The ethics of research related to healthcare in developing countries: a follow-up discussion paper (2005) [online]. 2002. [cited 2015 Jan 16]; Available from: http:// nuffieldbioethics.org/wp-content/uploads/2014/07/ HRRDC_Follow-up_Discussion_Paper.pdf

5. Council for International Organizations of Medical Sciences (CIOMS) in collaboration with the World Health Organization (WHO). International Ethical Guidelines for Biomedical Research Involving Human Subjects. Geneva: CIMOS; 2002. Available from: http://www.cioms.ch/publications/guidelines/ guidelines_nov_2002_blurb.htm

6. European Group on Ethics in Science and New Technologies. Opinion Nr 17 on ethical aspects of clinical research in developing countries. Luxembourg: Office for Official Publications of the European Communities; 2003. Available from: http://bookshop. europa.eu/en/opinion-nr-17-on-ethical-aspects-ofclinical-research-in-developing-countries-pbKA 5103601/

7. International Committee of Medical Journal Editors. Recommendations for the conduct, reporting, editing, and publication of scholarly work in medical journals: updated August 2013 [online]. 2013. [cited 2013 Aug 27]; Available from: http://www.icmje.org/icmjerecommendations.pdf

8. U.S. Department of Health and Human Services (DHHS) regulations for the protection of human subjects (45 CFR part 46) [online]. 2009. [cited $14 \mathrm{Nov}$ 28]; Available from: http://www.hhs.gov/ohrp/ policy/ohrpregulations.pdf

9. International Conference on Harmonisation (ICH) Harmonised Tripartite Guideline for Good Clinical Practice E6(R1) 1996 [online]. [cited 2015 Jan 28]; Available from: http://www.ich.org/products/ guidelines/efficacy/efficacy-single/article/goodclinical-practice.html

10. Robinson JK, Bhatia AC, Callen JP. Protection of patients' right to privacy in clinical photographs, video, and detailed case descriptions. JAMA Dermatol. 2014; 150:14-6. [editorial].

11. Slue WE Jr. Unmasking the lone ranger. N Engl J Med. 1989; 321:550-1.

12. Riis P, Nylenna M. Patients have a right to privacy and anonymity in medical publication. JAMA. 1991; 265:2720. 
13. Iverson C, Christiansen S, Flanagin A, et al. Protecting research participants' and patients' rights in scientific publication. In: Iverson C, Christiansen S, Flanagin A, et al., eds. AMA manual of style: a guide for authors and editors. 10th ed. New York: Oxford University Press; 2007.
14. International Committee of Medical Journal Editors. Protection of patients' rights to privacy. BMJ. 1995; 311:1272.

15. Snider DE. Patient consent for publication and the health of the public. JAMA. 1997; 278:624-6. 